\title{
Quantitative Analysis of Isolated Single-Wall Carbon Nanotubes with Their Molar Absorbance Coefficients
}

\author{
Shota Kuwahara, ${ }^{1,2}$ Yuki Kuwahara, ${ }^{1,3}$ and Hisanori Shinohara ${ }^{1}$ \\ ${ }^{1}$ Department of Chemistry, Nagoya University, Nagoya 464-8602, Japan \\ ${ }^{2}$ Department of Applied Chemistry, Chuo University, Tokyo 112-8551, Japan \\ ${ }^{3}$ Nanotube Research Center, National Institute of Advanced Industrial Science and Technology, Ibaraki 305-8565, Japan \\ Correspondence should be addressed to Shota Kuwahara; kuwahara@kc.chuo-u.ac.jp \\ and Hisanori Shinohara; noris@nagoya-u.jp
}

Received 14 April 2014; Accepted 28 July 2014; Published 12 August 2014

Academic Editor: Keita Kobayashi

Copyright (C) 2014 Shota Kuwahara et al. This is an open access article distributed under the Creative Commons Attribution License, which permits unrestricted use, distribution, and reproduction in any medium, provided the original work is properly cited.

The molar absorbance coefficients of metallic, semiconducting, and $(6,5)$ chirality enriched single-wall carbon nanotubes were evaluated by a spray technique combined with atomic force microscopy. Single-wall carbon nanotubes with isolated and a single predominant electronic type were obtained by using the density-gradient ultracentrifugation technique. In the visible region, all coefficients had similar values around $2-5 \times 10^{9} / \mathrm{mL} \mathrm{mol}^{-1} \mathrm{~cm}^{-1}$, independent of their diameter distribution and the electronic types of single-wall carbon nanotubes, and the $\varepsilon_{\mathrm{S} 22} / \varepsilon_{\mathrm{M} 11}$ and $\varepsilon_{\mathrm{S} 11} / \varepsilon_{\mathrm{M} 11}$ were estimated to be 1.0 and 4.0 , respectively. The coefficient strongly depends on the length of single-wall carbon nanotubes, independent of their electronic types and chirality.

\section{Introduction}

The absorption spectra of single-wall carbon nanotubes (SWCNTs) are one of the basic methods for determining the optical properties of SWCNTs and a very powerful tool for studying their electronic structure, purity, and quantity, known as purity assessment [1-5]. All current methods for producing SWCNTs, however, give mixtures of metallic (M-) and semiconducting (S-) SWCNTs with a variety of lengths and chiralities. This structural diversity complicates the quantitative characterization or purity assessment of the electronic types, amount, and morphology of SWCNTs in the materials.

Recent progress in the sonication techniques [6] allows us to obtain well-dispersed SWCNT solutions, which show electronic absorption spectra with very sharp peaks related to individual SWCNT structures. These advanced sonication techniques have been applied to separation of SWCNTs, and the scalable technique of the density-gradient ultracentrifugation (DGU) and column chromatography easily provides bulk quantities of SWCNTs with an individual and a single predominant electronic type [7-15]. By using these separated samples, the metal-to-semiconductor ratios $[10,16-18]$ were successfully evaluated.

In this study, a spray technique combined with atomic force microscopy (AFM) [5] was used to determine the molar absorbance coefficients of individual and single electronic types of SWCNT solutions which were prepared by DGU separation of SWCNTs $[9,10,12]$. The length-diameter distribution was statistically evaluated with AFM, and each of the obtained coefficients was fairly compared. Then, the metalto-semiconductor ratios in unsorted dispersed solutions were evaluated by a weighted sum of the spectra of M- and SSWCNTs in the visible region, which corresponds to $S_{11}, S_{22}$, $\mathrm{S}_{33}, \mathrm{M}_{11}$, and $\mathrm{M}_{22}$ [10]. The molar absorbance coefficient in UV region was also evaluated, and the length dependence on the coefficient of SWCNTs was discussed.

\section{Experimental}

In this study, different types of SWCNTs were used: HiPco (Carbon Nanotechnologies Inc.; average diameter $d_{t}$ : $\sim 1.0 \mathrm{~nm}$ ) [19] and CoMoCAT (Southwest Nanotechnologies; $d_{t}: \sim 0.8 \mathrm{~nm}$ ) [20]. $1 \mathrm{mg}$ of prepared SWCNTs was dispersed 


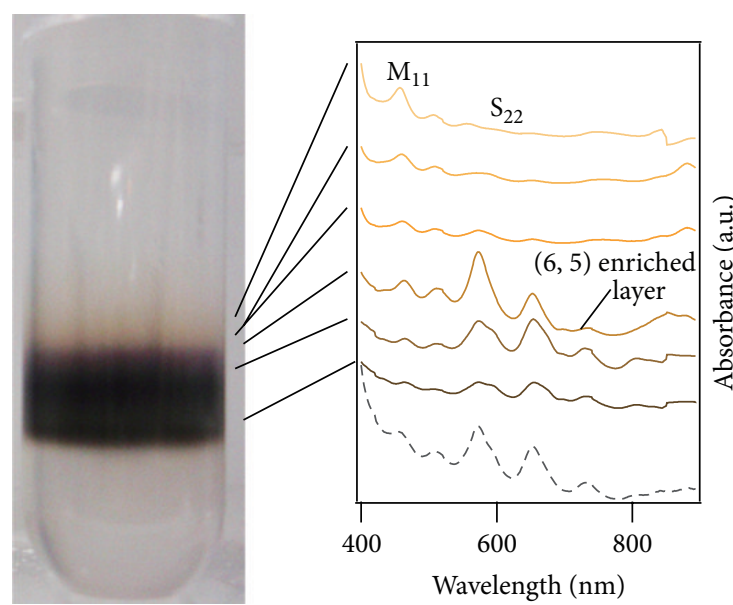

(a)

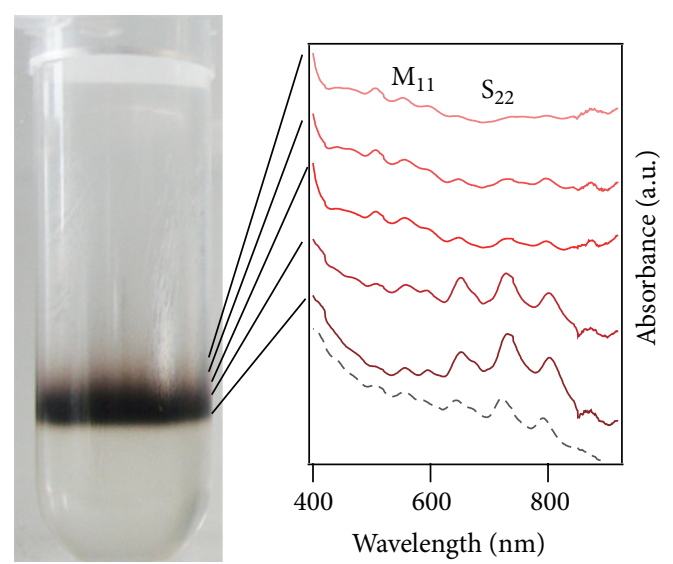

(b)

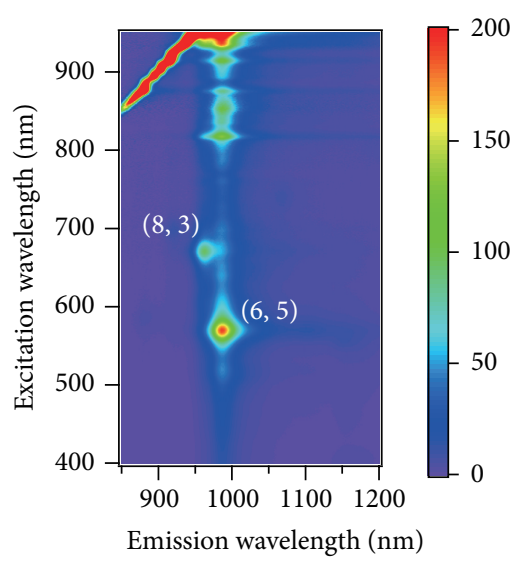

(d)

Figure 1: Photographs of the ultracentrifuge tubes of (a) CoMoCAT, (b) HiPco, and (c) (6,5) chirality-enriched samples after DGU. Each layer was collected from gradients at the points labeled by black lines and characterized by using optical absorbance. The dashed gray lines indicate unsorted materials. (d) A photoluminescence map for the $(6,5)$ chirality-enriched sample in (c). The red stripe of high intensity is due to Rayleigh scattered excitation light.

in $10 \mathrm{~mL}$ of $\mathrm{H}_{2} \mathrm{O}$ with 1.6 weight per volume (w/v) sodium cholate hydrate (SC; Sigma-Aldrich) and $0.4 \mathrm{w} / \mathrm{v}$ sodium deoxycholate (DOC; Tokyo Chemical Industry Co., Ltd.) as a cosurfactant using a $20 \mathrm{~W}$ probe tip ultrasonicator (Branson, Sonifier 250) for $20 \mathrm{~h}[9,21]$. During sonication, the solutions were immersed in a water bath to prevent heating. To remove large bundles, the solutions were centrifuged (Hitachi Koki, Himac CS100 GXL) at 250,000 $\mathrm{g}$ (74 krpm) for $1 \mathrm{~h}$ by using a fixed angle S100AT6 rotor (Hitachi Koki). The upper $80 \%$ of the supernatant was used for the separation.

By using the protocol for DGU separation of SWCNTs reported by Arnold et al. [12], (6,5) chirality-enriched samples, M- and S-SWCNTs, were prepared. To improve the separation yield, the surfactant concentration was optimized to increase the distance between $\mathrm{M}$ - and S-SWCNTs enriched layers in the centrifugation tube $[9,10,22]$.

Then, iodixanol, sodium cholate, and deoxycholate sodium salts were removed from obtained samples through dialysis [23] in 1\% w/v aqueous SDS using a visking membrane (MW: $\sim 12,000$, cellulose tube). Absorption spectra of the dispersions were measured, and the samples were sprayed onto $\mathrm{SiO}_{2}$ substrates for AFM evaluation [5]. Each sample was accurately diluted using $1 \% \mathrm{w} / \mathrm{v}$ SDS- $\mathrm{H}_{2} \mathrm{O}$, and $2 \mathrm{~mL}$ solution was prepared. The sprayed samples were observed by AFM to determine the number of SWCNTs in dispersed solutions, and their absorbance coefficients were calculated as previously reported [5]. All AFM measurements were carried out on Dimension 3100, Nanoscope IV (Veeco, Digital Instruments) in tapping mode with $\mathrm{Si}$ cantilevers at room temperature.

The $(6,5)$ chirality-enriched sample was also characterized by the photoluminescence (PL) measurement using a Shimadzu NIR-PL system (CNT-RF) equipped with a liquid $\mathrm{N}_{2}$-cooled InGaAs detector array. A $10 \mathrm{~nm}$ slit width was used for both excitation and emission. Excitation light was generated by a xenon arc lamp with an exposure time of $5 \mathrm{sec}$. 


\section{Results and Discussion}

The photographs of the centrifuge tubes and optical absorbance spectra after DGU are shown in Figure 1. By comparing the diameter distribution of the samples with the calculated energies between mirror-image spikes in the density of states, each absorbance was assigned as $S_{22}$ and $\mathrm{M}_{11}$ as denoted in the spectra [24]. The absorption spectra of M-SWCNTs showed the strong suppression of optical transitions associated with S-SWCNT species and vice versa. In the DGU separation of CoMoCAT, a $(6,5)$ chirality-enriched layer, the purple-colored layer, appeared between the M- and S- SWCNT layers (Figure 1(a)) [11, 12].

The relative magnitude of the absorbance of $\mathrm{M}$ CoMoCAT and S-CoMoCAT varied with the concentration of cosurfactants in the sample layers. At a total amount of $3.5 \% \mathrm{w} / \mathrm{v}$ surfactant, the strong suppression of the absorption of S-CoMoCAT was observed, while M-HiPco was well separated at a total amount of $2.0 \mathrm{w} / \mathrm{v}$ surfactant. This indicates that a high concentration of surfactants was needed to separate SWCNTs with small diameters, CoMoCAT, by their electronic types. It is suggested that the coordination and the mass per volume of a wrapped and hydrated SWCNT are important for separation by DGU during the first step of diameter separation and that the interaction of surfactants within gradients and coordinated around SWCNTs is more effective for separations when the gradients are slowly sedimented towards the bottom of the centrifuge tube. The SWCNTs with small diameters have less $\pi$ interactions and fewer differences in mass per volume of a wrapped and hydrated nanotube rather than SWCNTs with larger diameter due to the difference in sidewall curvature and surfactant coordination around SWCNTs. As a consequence, in order to use the interaction for sorting with the electronic type of SWCNTs, it is suggested that a high concentration of surfactant in the gradient is required.

The absorption spectrum from the $(6,5)$ chiralityenriched sample (Figure 1(c)) showed strong absorption signals at a wavelength of $570 \mathrm{~nm}$ and $987 \mathrm{~nm}$. A spectrum of unsorted supernatant from the starting material is also shown in Figure 1(c) for comparison. The absorption peak at $570 \mathrm{~nm}$ and $987 \mathrm{~nm}$ was assigned as $S_{22}$ and $S_{11}$ in the $(6,5)$ tube, respectively. The other partially overlapped absorption around a wavelength of $960 \mathrm{~nm}$ is associated with $\mathrm{S}_{22}$ in the $(8,3)$ tubes. The PL mapping of the $(6,5)$ chirality-enriched layer (Figure 1(d)) also indicated that PL from the $(6,5)$ chirality was significantly enhanced, and the low intensity of PL from $(8,3)$ chirality was observed too.

The number of SWCNTs in the separated samples and their absorbance coefficients were determined as previously reported [5]. Briefly, monolayer SDS membranes were formed by spraying dispersed SWCNTs onto $\mathrm{SiO}_{2}$ substrates, and the area of the formed membranes was evaluated by AFM observation (Figure 2(f)). Then, the SDS molecules alone were washed out with rinsing in water, and SWCNTs were observed by AFM (Figure 2(g)). Finally, by comparing the number of SDS molecules and SWCNTs in the formed membranes, the molarity of SWCNTs in 1\% w/v SDS dispersion was accurately determined, and the molar absorbance coefficient was evaluated.

In the case of the $(6,5)$ chirality-enriched sample (diameter, $d=0.76 \mathrm{~nm}$ ), the average number of SWCNTs in a bundle and the mean length was estimated to be $1.1 \mathrm{~nm}$ and $350 \mathrm{~nm}$ from the height and length distribution (Figure 2(c)). The absorbance coefficient of the $(6,5)$ chirality-enriched SWCNTs was evaluated to be $6.9 \times$ $10^{-15} / \mathrm{mLSWCNT}^{-1} \mathrm{~cm}^{-1}$ at a wavelength of $570 \mathrm{~nm}$ and $1.9 \times 10^{-14} / \mathrm{mLSWCNT}^{-1} \mathrm{~cm}^{-1}$ at a wavelength of $987 \mathrm{~nm}$, respectively. Moreover, a SWCNT with the diameter of $0.76 \mathrm{~nm}$ and the length of $350 \mathrm{~nm}$ was regarded as an individual molecule, and the molar absorbance coefficients of the $(6,5)$ chirality-enriched SWCNT were estimated to be $4.2 \times 10^{9} / \mathrm{mL} \mathrm{mol}^{-1} \mathrm{~cm}^{-1}$ at a wavelength of $570 \mathrm{~nm}$ and $1.1 \times 10^{10} / \mathrm{mL} \mathrm{mol}^{-1} \mathrm{~cm}^{-1}$ at $987 \mathrm{~nm}$.

We also obtained the molar absorbance coefficients of each SWCNT dispersed solutions separated by their electronic types. Figure 3 presents the optical absorption spectra of each sample normalized by their determined absorbance coefficients. In the visible region, all coefficients had almost the same value of $2-5 \times 10^{9} / \mathrm{mL} \mathrm{mol}^{-1} \mathrm{~cm}^{-1}$ independent of their diameter distributions and electronic types of SWCNT, and $\varepsilon_{\mathrm{S} 22} / \varepsilon_{\mathrm{M} 11}$ was estimated to be $\sim 1.0$, while $\varepsilon_{\mathrm{S} 11} / \varepsilon_{\mathrm{M} 11}$ was estimated to be $\sim 4.0$. It is indicated that the obtained absorption coefficients enable us to estimate the relative concentration of SWCNTs species with their electronic types or chiralities.

To evaluate the metal-to-semiconductor ratio in the starting materials, the spectra of the unsorted dispersed solutions were reproduced by the sum spectra of the M- and S-SWCNTs [10]. As shown in Figure 4(a), the sum spectrum of the M-HiPco multiplied by 0.3 and the S-HiPco spectrum multiplied by 0.7 reproduced the unsorted spectrum. It is understood that the starting materials of HiPco contained $30 \%$ metal and $70 \%$ semiconductor. It is well known that the concentration of M-SWCNTs would be 33\% when all chiralities grow at the same rate. The value is very close to our evaluation as mentioned above.

In the case of CoMoCAT samples, the sum of the spectra of the M-, S-, and the $(6,5)$ chirality-enriched SWCNTs was required to reproduce the unsorted dispersed solution since a $(6,5)$ chirality-enriched layer existed between the layers of $\mathrm{M}$ - and S-SWCNTs during the sorting of CoMoCAT samples. The sum spectrum of the M-CoMoCAT multiplied by 0.2 , the S-CoMoCAT multiplied by 0.5 , and the $(6,5)$ chiralityenriched layer multiplied by 0.3 reproduced the spectrum of the unsorted solution as shown in Figure 4(b).

The molar absorbance coefficient of SWCNTs in the UV absorption region, known as the collective excitation of the $\pi$ electron system polarized along the nanotube axis, was previously reported and found to be ca. $1.9 \times 10^{7} / \mathrm{L} \mathrm{mol}^{-1} \mathrm{~cm}^{-1}$ at $280 \mathrm{~nm}$ with an average length of around $660 \mathrm{~nm}$ [5]. Figure 5 presents the optical absorption spectra in the UV region of 


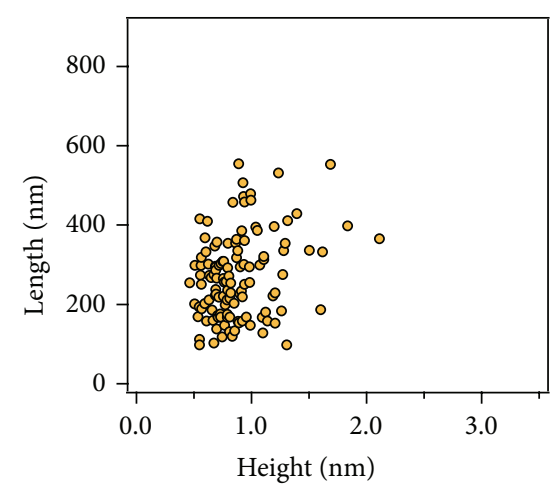

(a)

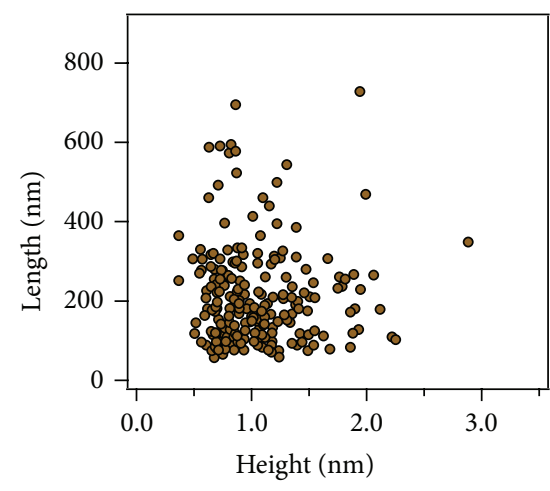

(b)

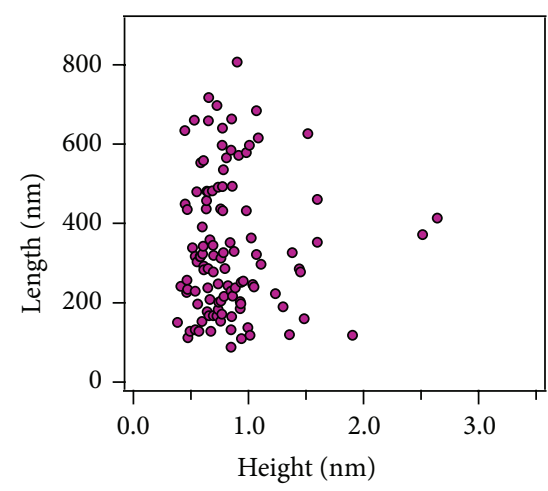

(c)

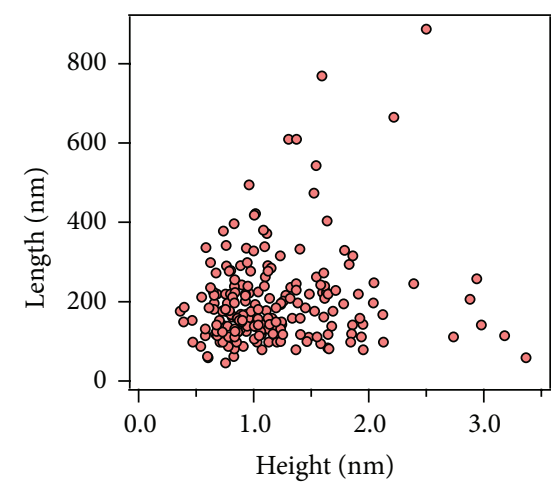

(d)

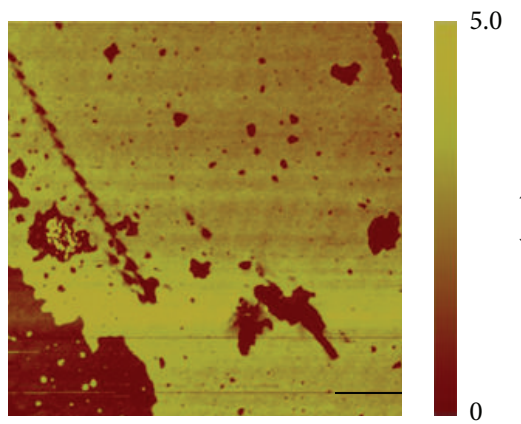

(f)

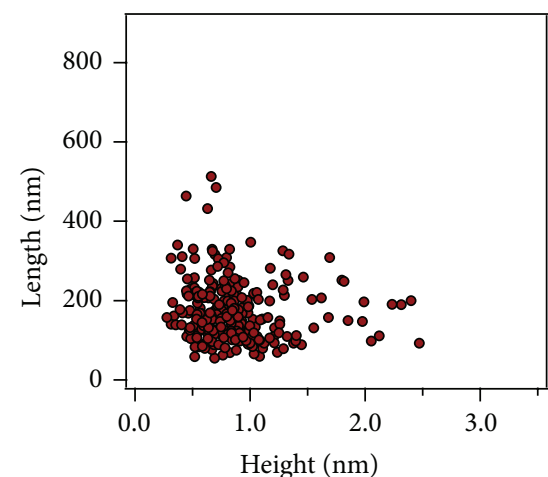

(e)

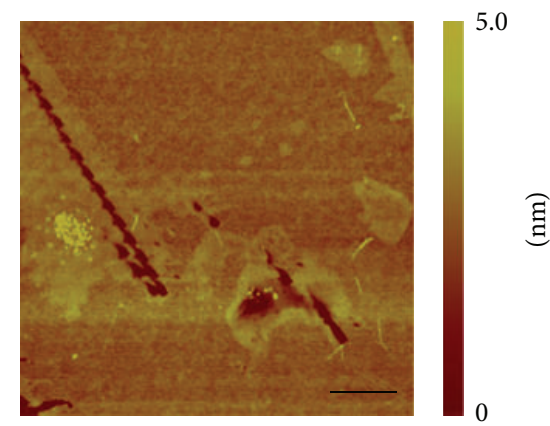

(g)

Figure 2: Height and length distributions of (a) M-CoMoCAT, (b) S-CoMoCAT, (c) the (6,5) chirality-enriched CoMoCAT, (d) M-HiPco, and (e) S-HiPco in $1 \% \mathrm{w} / \mathrm{v}$ aqueous SDS dispersions evaluated by AFM. AFM images of an S-CoMoCAT sample prepared by the spray technique, (f) before and (g) after rinsing with distilled water. Scale bar is $1 \mu \mathrm{m}$.

the HiPco samples in this study normalized by the determined absorbance coefficients. The value of the unsorted HiPco was obtained by the sum spectrum of the M-HiPco multiplied by 0.3 and the S-HiPco multiplied by 0.7 . The average length was around $180 \mathrm{~nm}$, and the molar absorbance coefficient was evaluated as $3.6 \times 10^{6} / \mathrm{L} \mathrm{mol}^{-1} \mathrm{~cm}^{-1}$ at $280 \mathrm{~nm}$ in this work. The value of the coefficient was about five times smaller than the one previously reported due to the shorter length of SWCNTs. It is understood that the molar absorbance coefficient is proportional to the average length of SWCNTs in the dispersed solutions and that the coefficient depends on the number of carbons in a SWCNT. The obtained molar absorbance coefficient normalized by their average length in this work, however, was a little smaller than the previously reported one. It is suggested that damage on the sidewall of SWCNTs generated by long-time and high power sonication process broke the crystalline of SWCNTs, which decreased the absorbance coefficient of short-length SWCNTs.

\section{Conclusion}

The molar absorbance coefficients of M- and S-SWCNTs, which were separated by using the DGU method, have been successfully evaluated by the spray technique we developed. 


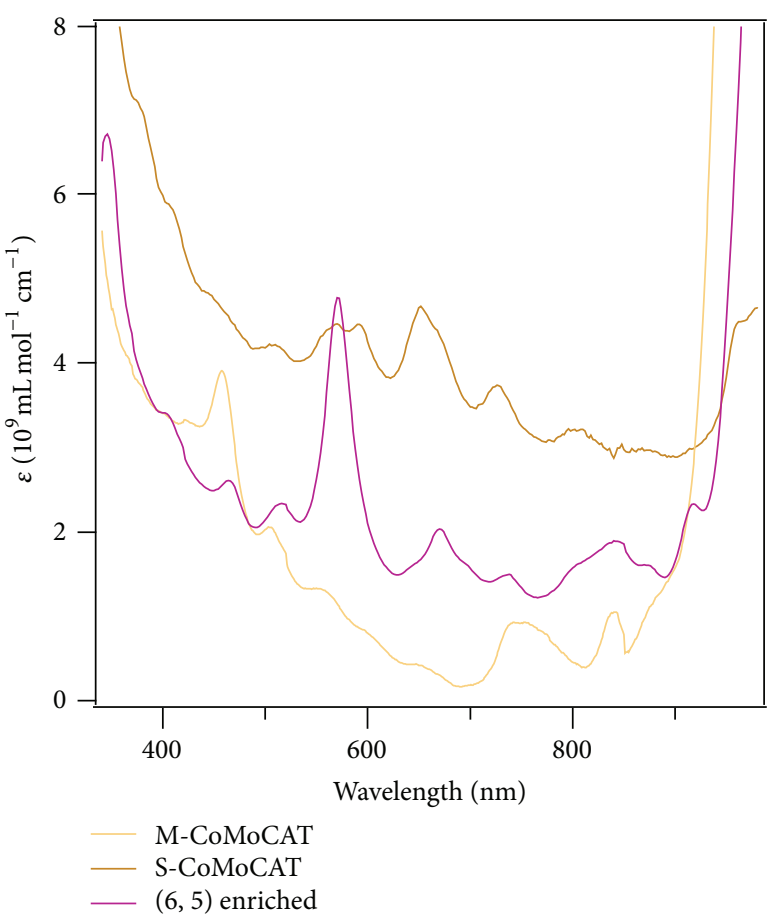

(a)

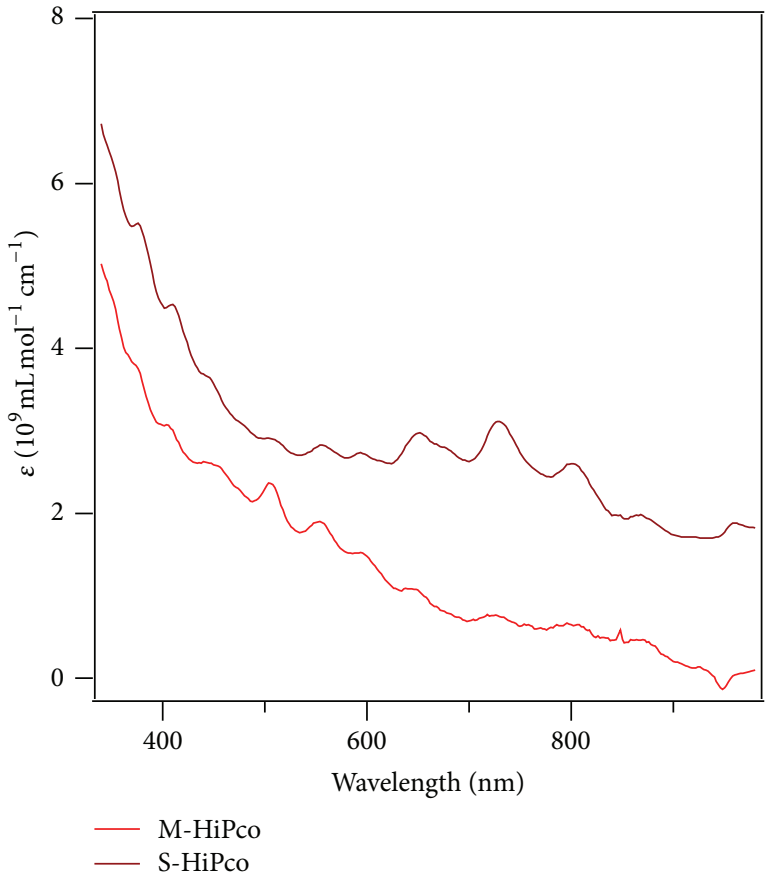

(b)

FIGURE 3: The optical absorption spectra of (a) CoMoCAT and (b) HiPco normalized by the determined molar absorbance coefficients.

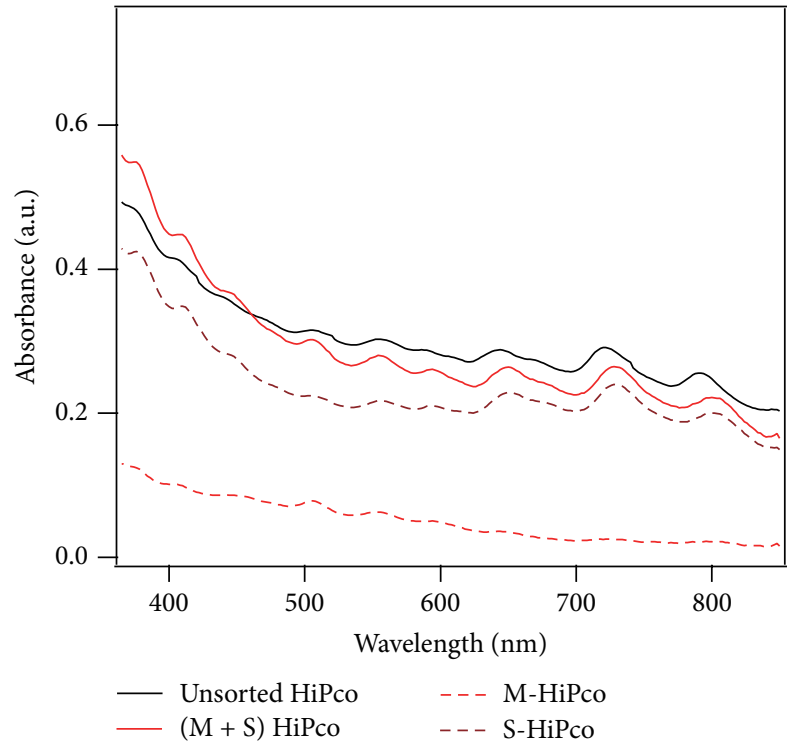

(a)

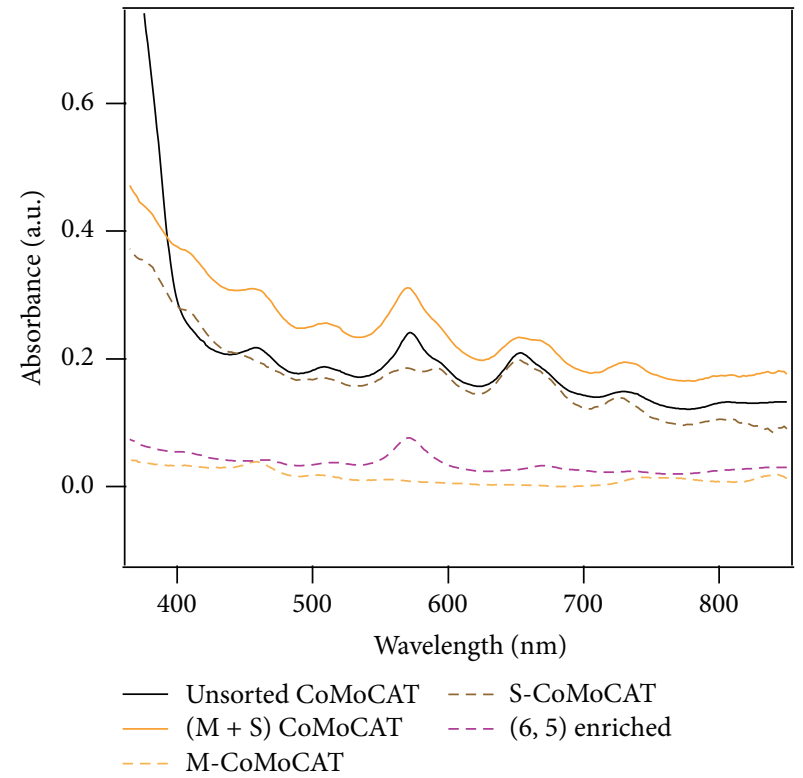

(b)

FIgURE 4: Optical absorption spectra of (a) HiPco and (b) CoMoCAT with the sum spectrum of the M- and S-SWCNTs.

In the visible region, all coefficients had similar values of $2-$ $5 \times 10^{9} / \mathrm{mL} \mathrm{mol}^{-1} \mathrm{~cm}^{-1}$, independent of their diameter distribution and the electronic type of SWCNTs, and $\varepsilon_{\mathrm{M} 11} / \varepsilon_{\mathrm{S} 22}$ was estimated to be $\sim 1.0$. The $(6,5)$ enriched samples had the same value. As a consequence, the absorbance coefficient of SWCNTs was independent of their electronic types and chiralities but was proportional to their length. It is suggested that we can assess the number of SWCNTs in dispersions when the length distributions of the dispersions are evaluated. 


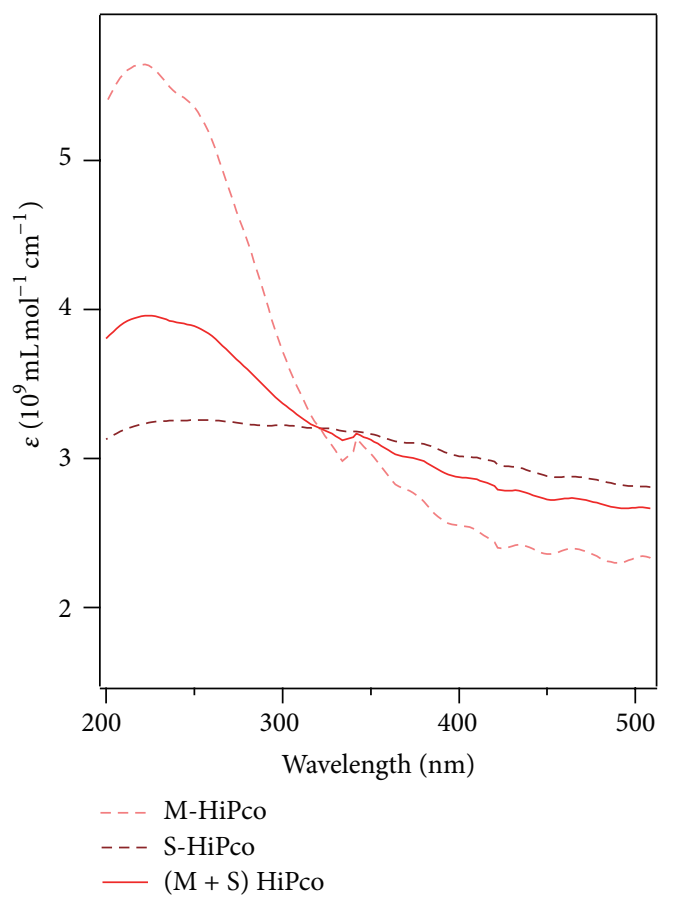

FIgURE 5: Optical absorption spectra of M- and S-HiPco normalized by the determined molar absorbance coefficients in UV region with the sum spectrum of the M- and S-SWCNTs.

\section{Conflict of Interests}

The authors declare that there is no conflict of interests regarding the publication of this paper.

\section{References}

[1] D. Nishide, Y. Miyata, K. Yanagi, T. Tanaka, and H. Kataura, "PERIPUTOS: purity evaluated by Raman intensity of pristine and ultracentrifuged topping of single-wall carbon nanotubes," Physica Status Solidi (B) Basic Research, vol. 246, no. 11-12, pp. 2728-2731, 2009.

[2] B. J. Landi, C. D. Cress, C. M. Evans, and R. P. Raffaelle, "Thermal oxidation profiling of single-walled carbon nanotubes," Chemistry of Materials, vol. 17, no. 26, pp. 6819-6834, 2005.

[3] B. J. Landi, H. J. Ruf, C. M. Evans, C. D. Cress, and R. P. Raffaelle, "Purity assessment of single-wall carbon nanotubes, using optical absorption spectroscopy," The Journal of Physical Chemistry B, vol. 109, no. 20, pp. 9952-9965, 2005.

[4] A. G. Ryabenko, T. V. Dorofeeva, and G. I. Zvereva, "UVVIS-NIR spectroscopy study of sensitivity of single-wall carbon nanotubes to chemical processing and Van-der-Waals SWNT/SWNT interaction. Verification of the SWNT content measurements by absorption spectroscopy," Carbon, vol. 42, no. 8-9, pp. 1523-1535, 2004.

[5] S. Kuwahara, T. Sugai, and H. Shinohara, "Determining exact molar absorbance coefficients of single-wall carbon nanotubes," Physical Chemistry Chemical Physics, vol. 11, no. 7, pp. 1091-1097, 2009.

[6] M. J. O'Connell, S. H. Bachilo, C. B. Huffman et al., "Band gap fluorescence from individual single-walled carbon nanotubes," Science, vol. 297, no. 5581, pp. 593-596, 2002.
[7] A. A. Green and M. C. Hersam, "Colored semitransparent conductive coatings consisting of monodisperse metallic singlewalled carbon nanotubes," Nano Letters, vol. 8, no. 5, pp. 1417$1422,2008$.

[8] M. S. Arnold, S. I. Stupp, and M. C. Hersam, "Enrichment of single-walled carbon nanotubes by diameter in density gradients," Nano Letters, vol. 5, no. 4, pp. 713-718, 2005.

[9] K. Yanagi, Y. Miyata, and H. Kataura, "Optical and conductive characteristics of metallic single-wall carbon nanotubes with three basic colors; cyan, magenta, and yellow," Applied Physics Express, vol. 1, no. 3, Article ID 034003, 2008.

[10] Y. Miyata, K. Yanagi, Y. Maniwa, and H. Kataura, "Optical evaluation of the metal-to-semiconductor ratio of single-wall carbon nanotubes," Journal of Physical Chemistry C, vol. 112, no. 34, pp. 13187-13191, 2008.

[11] J. Crochet, M. Clemens, and T. Hertel, "Quantum yield heterogeneities of aqueous single-wall carbon nanotube suspensions," Journal of the American Chemical Society, vol. 129, no. 26, pp. 8058-8059, 2007.

[12] M. S. Arnold, A. A. Green, J. F. Hulvat, S. I. Stupp, and M. C. Hersam, "Sorting carbon nanotubes by electronic structure using density differentiation.," Nature nanotechnology, vol. 1, no. 1, pp. 60-65, 2006.

[13] H. Liu, D. Nishide, T. Tanaka, and H. Kataura, "Large-scale single-chirality separation of single-wall carbon nanotubes by simple gel chromatography," Nature Communications, vol. 2, no. 1, article 309, 2011.

[14] T. Tanaka, Y. Urabe, D. Nishide, and H. Kataura, "Continuous separation of metallic and semiconducting carbon nanotubes using agarose gel," Applied Physics Express, vol. 2, no. 12, Article ID 125002, 2009.

[15] K. Moshammer, F. Hennrich, and M. M. Kappes, "Selective suspension in aqueous sodium dodecyl sulfate according to electronic structure type allows simple separation of metallic from semiconducting single-walled carbon nanotubes," Nano Research, vol. 2, no. 8, pp. 599-606, 2009.

[16] Y. Li, D. Mann, M. Rolandi et al., "Preferential growth of semiconducting single-walled carbon nanotubes by a plasma enhanced CVD method," Nano Letters, vol. 4, no. 2, pp. 317-321, 2004.

[17] W. Kim, H. C. Choi, M. Shim, Y. Li, D. Wang, and H. Dai, "Synthesis of ultralong and high percentage of semiconducting single-walled carbon nanotubes," Nano Letters, vol. 2, no. 7, pp. 703-708, 2002.

[18] A. Jorio, A. P. Santos, H. B. Ribeiro et al., "Quantifying carbonnanotube species with resonance Raman scattering," Physical Review B-Condensed Matter and Materials Physics, vol. 72, no. 7, Article ID 075207, 2005.

[19] P. Nikolaev, M. J. Bronikowski, R. K. Bradley et al., "Gasphase catalytic growth of single-walled carbon nanotubes from carbon monoxide," Chemical Physics Letters, vol. 313, no. 1-2, pp. 91-97, 1999.

[20] B. Kitiyanan, W. E. Alvarez, J. H. Harwell, and D. E. Resasco, "Controlled production of single-wall carbon nanotubes by catalytic decomposition of CO on bimetallic Co-Mo catalysts," Chemical Physics Letters, vol. 317, no. 3-5, pp. 497-503, 2000.

[21] W. Wenseleers, I. L. Vlasov, E. Goovaerts, E. D. Obraztsova, A. S. Lobach, and A. Bouwen, "Efficient isolation and solubilization of pristine single-walled nanotubes in bile salt micelles," Advanced Functional Materials, vol. 14, no. 11, pp. 1105-1112, 2004. 
[22] Y. Miyata, K. Yanagi, Y. Maniwa, and H. Kataura, "Highly stabilized conductivity of metallic single wall carbon nanotube thin films," The Journal of Physical Chemistry C, vol. 112, no. 10, pp. 3591-3596, 2008.

[23] A. A. Green and M. C. Hersam, "Ultracentrifugation of singlewalled nanotubes," Materials Today, vol. 10, no. 12, pp. 59-60, 2007.

[24] H. Kataura, Y. Kumazawa, Y. Maniwa et al., "Optical properties of single-wall carbon nanotubes," Synthetic Metals, vol. 103, no. 1-3, pp. 2555-2558, 1999. 

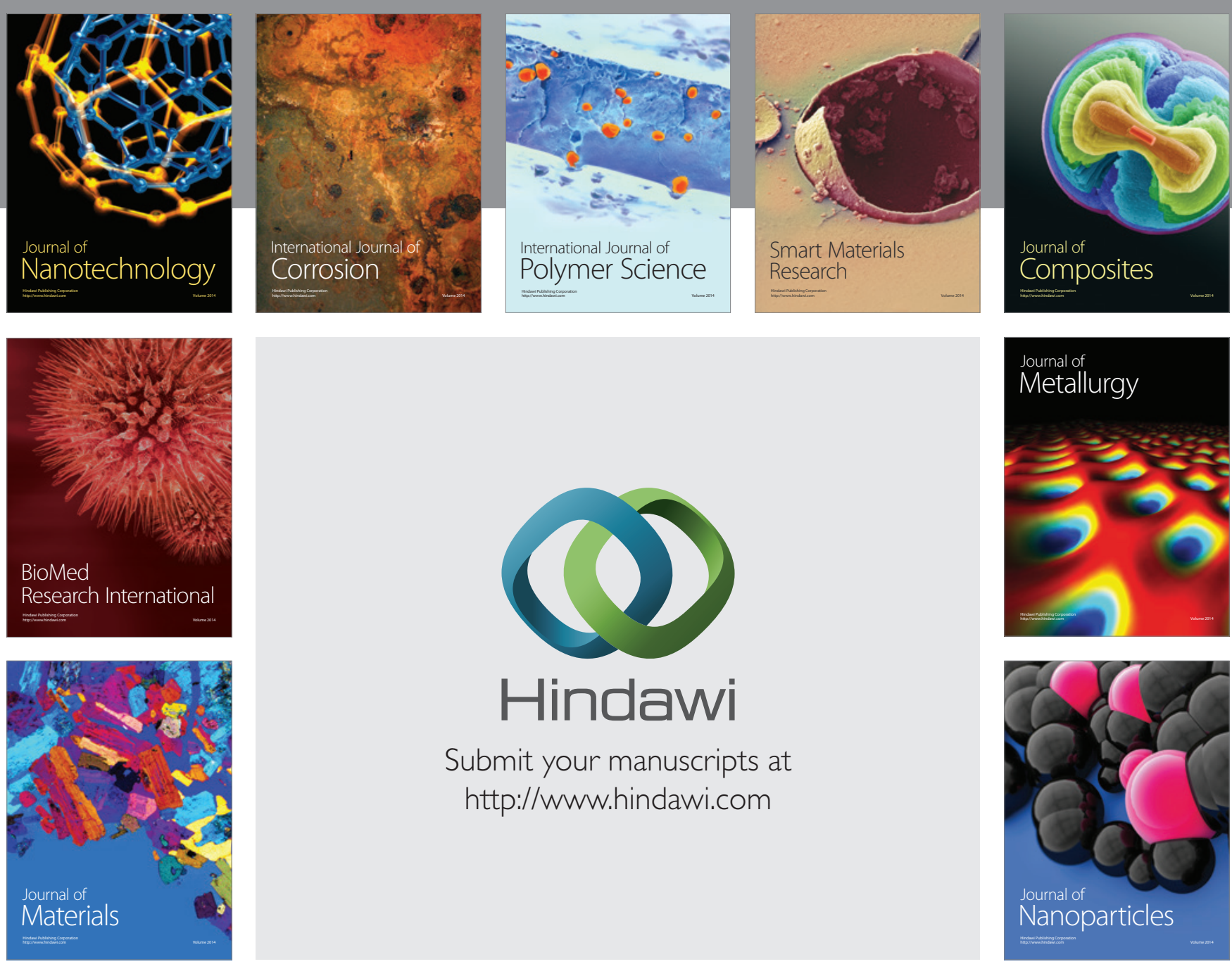

Submit your manuscripts at http://www.hindawi.com
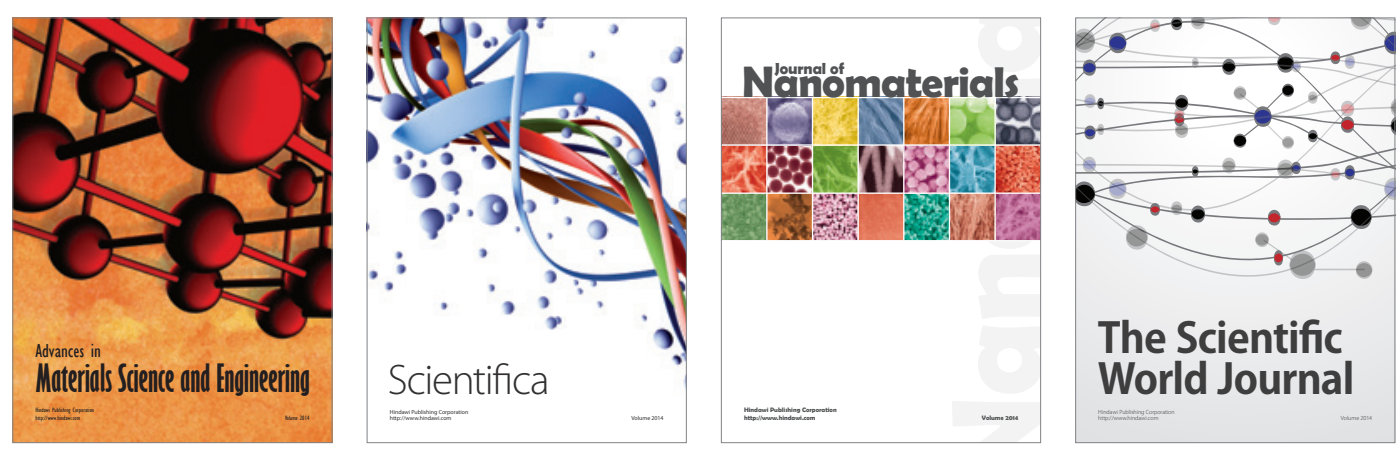

\section{The Scientific World Journal}
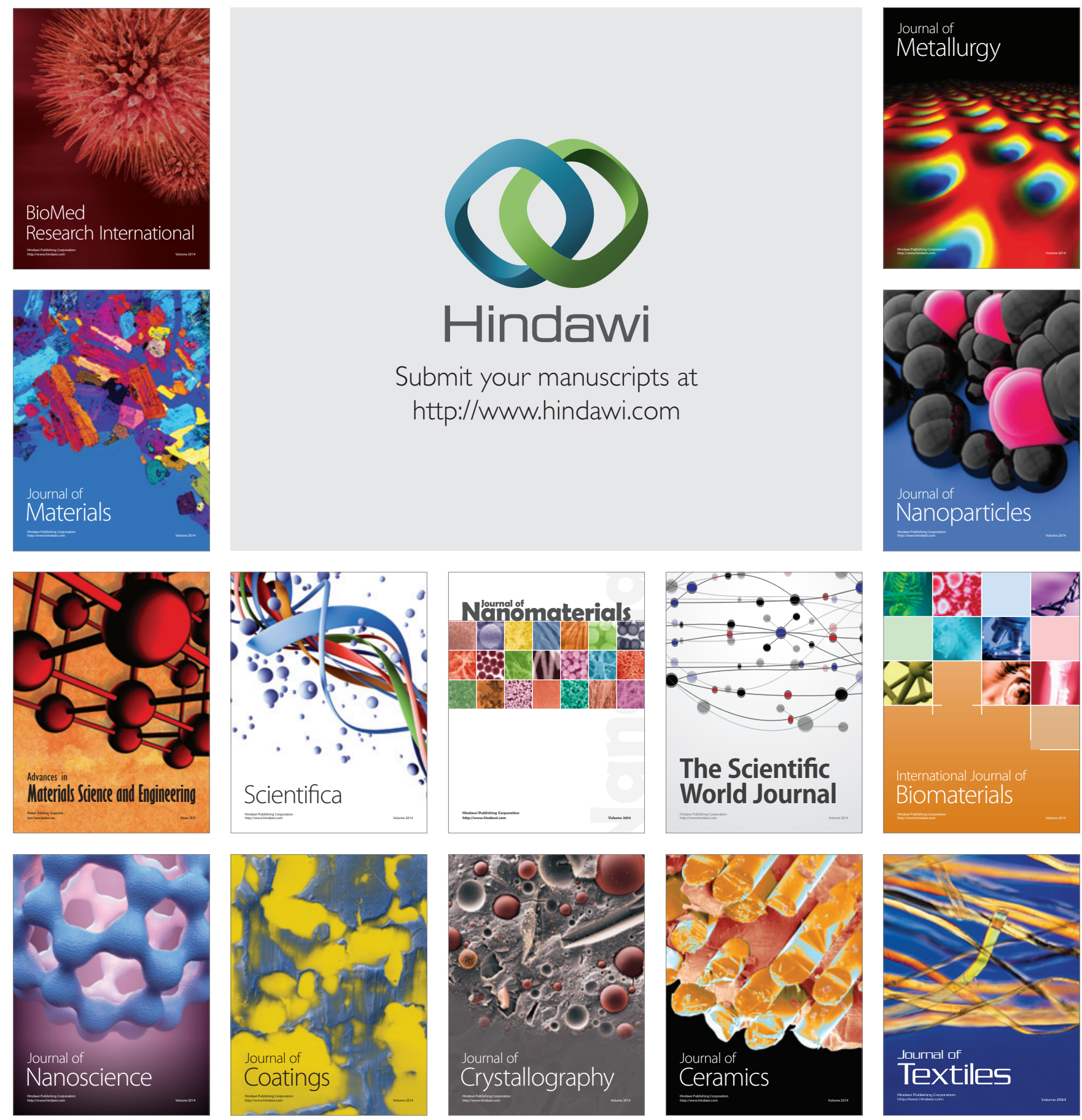